\title{
Uninsured Medical Expense
}

National Cancer Institute

\section{Source}

National Cancer Institute. Uninsured Medical Expense. NCI Thesaurus. Code C17718.

Uninsured Medical Expense; payment for the uninsured portion of regular medical expenses, such as co-payments, deductibles, and uncovered expenses. Uninsured medical expense encompasses a range of items that includes co-payments, medicine costs, uncovered procedures and conditions, as well as cash payments in lieu of health insurance. 